\title{
Diagnosis of Gastric Subepithelial Tumors Using Endoscopic Ultrasonography or Abdominopelvic Computed Tomography: Which is Better?
}

\author{
Eun Young Park and Gwang Ha Kim \\ Department of Internal Medicine, Pusan National University School of Medicine and Biomedical Research Institute, Pusan National University \\ Hospital, Busan, Korea.
}

See "Comparison of the Diagnostic Ability of Endoscopic Ultrasonography and Abdominopelvic Computed Tomography in the Diagnosis of Gastric Subepithelial Tumors” by Sang Yoon Kim, Ki-Nam Shim, Joo-Ho Lee, et al., on page 565-573.

Gastric subepithelial tumors (SETs) are predominantly asymptomatic lesions with normal overlying mucosa and are often found incidentally during endoscopic or radiologic examinations. Depending on the histopathological type, SETs may arise from the deep mucosa to the serosa of the stomach. The exact incidence of gastric SETs is unknown. However, according to the Korea EUS Study Group (unpublished data), the prevalence of gastric SETs, detected during routine esophagogastroduodenoscopy, in Korea is 3.1\% (2,685/87,578 individuals). Gastric SETs include various types of tumors that can largely be classified as either benign or malignant lesions. Benign lesions include lipoma, leiomyoma, varices, ectopic pancreas, duplication cyst, and inflammatory fibroid polyp, whereas malignant lesions include gastrointestinal stromal tumor (GIST), neuroendocrine tumor, lymphoma, and rarely metastasis. ${ }^{1,2}$

The differential diagnosis of gastric SETs begins with meticulous endoscopic examination, where the size, shape, color,

Received: September 14, 2019 Accepted: October 10, 2019

Correspondence: Gwang Ha Kim

Department of Internal Medicine, Pusan National University School of Medicine and Biomedical Research Institute, Pusan National University Hospital, 179 Gudeok-ro, Seo-gu, Busan 49241, Korea

Tel: +82-51-240-7869, Fax: +82-51-244-8180, E-mail: doc0224@pusan.ac.kr ORCID: https://orcid.org/0000-0001-9721-5734

(c) This is an Open Access article distributed under the terms of the Creative Commons Attribution Non-Commercial License (http://creativecommons.org/ licenses/by-nc/3.0) which permits unrestricted non-commercial use, distribution, and reproduction in any medium, provided the original work is properly cited. mobility, consistency, pulsation, and presence of erosion or ulcer in the overlying mucosa are observed. In general, SETs have a normal-appearing mucosa; the presence of erythema or ulcer is associated with the risk of increase in SET size. Several signs such as the rolling, pillow, naked fat, and tenting signs can be confirmed by biopsy forceps manipulation of the SETs, which are useful for the differential diagnosis. Endoscopic ultrasonography (EUS) is the best imaging modality for evaluating gastric SETs. It can assess the accurate size, layer of origin, margin status, echogenicity, homogeneity, and presence of echogenic foci in the lesion. ${ }^{1,2}$ Abdominopelvic computed tomography (CT) is also helpful for evaluating gastric SETs because it is less invasive and has several advantages, such as assessing the involvement of the gastric wall and adjacent structures including lymph nodes and the extragastric extent of the lesion. However, it cannot evaluate the layer of origin., Management depends on the histopathological type of gastric SETs; however, both EUS and abdominopelvic CT are not confirmative modalities for diagnosing gastric SETs. Therefore, pathologic confirmation is occasionally needed. ${ }^{5}$ There are several methods for pathologic diagnosis, including bite-on-bite biopsy, EUS-guided fine-needle aspiration, EUS-guided fine-needle biopsy, and even endoscopic mucosal resection or endoscopic submucosal dissection. ${ }^{6,7}$

In this issue of Clinical Endoscopy, Kim et al. ${ }^{8}$ compared the diagnostic ability of EUS and abdominopelvic CT and assessed their accuracies for diagnosing gastric SETs based on 
surgical histopathology results. The study included a total of 53 cases of gastric SETs, which were evaluated by both EUS and abdominopelvic $\mathrm{CT}$ before laparoscopic wedge resection. The median size of the gastric SETs was $3 \mathrm{~cm}$, and there was no difference in size between the malignant/potentially malignant and benign lesions. The overall diagnostic accuracies of EUS and abdominal CT were $64.2 \%$ and $50.9 \%$, respectively $(p=0.238)$. However, there was no significant difference in the diagnostic accuracy between EUS and abdominopelvic CT for diagnosing both malignant/potentially malignant lesions and benign lesions ( $p=0.762$ and $p=0.160$, respectively). For malignant/potentially malignant gastric SETs, EUS and abdominopelvic CT showed relatively high sensitivities and low specificities of $81.3 \%$ and $75.0 \%$ and $38.1 \%$ and $14.3 \%$, respectively. The overall accuracy of EUS for gastric SETs was $64.2 \%$, which is consistent with the results of previous studies reporting an accuracy of $46 \%-67 \%{ }^{2,3,7}$ The overall accuracy of abdominopelvic CT (50.9\%) was lower than that of EUS. Both EUS and abdominopelvic CT demonstrated good feasibility for detection of GISTs; however, EUS was more reliable for leiomyoma and ectopic pancreas, whereas abdominopelvic CT was more acceptable for lipoma and duplication cyst.

This study was a retrospective, single-center study and included only surgically resected gastric SETs. Inclusion of more endoscopically resected SETs or SETs diagnosed with EUS or $\mathrm{CT}$ alone without histopathologic confirmation may affect the results. Additionally, the SETs included in this study were relatively large (median size: $3 \mathrm{~cm}$ ). In a previous study, abdominopelvic CT showed good feasibility for depiction of gastric SETs $>10 \mathrm{~mm} .{ }^{4}$ However, several studies have reported that EUS is the gold standard for the diagnosis and detection of gastric SETs, and abdominopelvic CT cannot be an alternative to EUS, especially for small SETs. ${ }^{3}$ The relatively high accuracy of abdominopelvic CT may be attributed the large size of gastric SETs in this study. Most gastric SETs are asymptomatic and small in size $(<2 \mathrm{~cm})$, and only $3.2 \%-8.5 \%$ of gastric SETs have been reported to increase in size. ${ }^{9}$ Therefore, it would not be feasible to evaluate most gastric SETs using abdominopelvic CT. In addition, if the radiologists were not blind to the EUS findings of gastric SETs, the diagnostic accuracy of abdominopelvic CT could have been improved through a comprehensive interpretation of both modalities. ${ }^{10}$
However, this study has reasonably demonstrated that abdominopelvic CT is a feasible diagnostic modality for malignant/potentially malignant gastric SETs such as GIST or lymphoma. Abdominopelvic CT is also complementary in evaluating benign lesions, such as lipoma and duplication cyst. With the increasing detection of gastric SETs in screening endoscopy, a more adequate evaluation modality should be determined. Therefore, further studies with a large number of SETs, including small lesions, should be conducted.

Conflicts of Interest

The authors have no financial conflicts of interest.

ORCID

Eun Young Park: https://orcid.org/0000-0003-4933-7790

\section{REFERENCES}

1. Hwang JH, Rulyak SD, Kimmey MB. American Gastroenterological Association Institute technical review on the management of gastric subepithelial masses. Gastroenterology 2006;130:2217-2228.

2. Sakamoto H, Kitano M, Kudo M. Diagnosis of subepithelial tumors in the upper gastrointestinal tract by endoscopic ultrasonography. World J Radiol 2010;2:289-297.

3. Goto O, Kambe H, Niimi K, et al. Discrepancy in diagnosis of gastric submucosal tumor among esophagogastroduodenoscopy, CT, and endoscopic ultrasonography: a retrospective analysis of 93 consecutive cases. Abdom Imaging 2012;37:1074-1078.

4. Ra JC, Lee ES, Lee JB, et al. Diagnostic performance of stomach CT compared with endoscopic ultrasonography in diagnosing gastric subepithelial tumors. Abdom Radiol (NY) 2017;42:442-450.

5. Cho JW. Current guidelines in the management of upper gastrointestinal subepithelial tumors. Clin Endosc 2016;49:235-240.

6. Shin SY, Lee SJ, Jun JH, et al. Mucosal incision and forceps biopsy for reliable tissue sampling of gastric subepithelial tumors. Clin Endosc 2017;50:64-68.

7. Moon JS. Role of endoscopic ultrasonography in guiding treatment plans for upper gastrointestinal subepithelial tumors. Clin Endosc 2016;49:220-225.

8. Kim SY, Shim KN, Lee JH, et al. Comparison of the diagnostic ability of endoscopic ultrasonography and abdominopelvic computed tomography in the diagnosis of gastric subepithelial tumors. Clin Endosc 2019;52:565-573.

9. Kim MY, Jung HY, Choi KD, et al. Natural history of asymptomatic small gastric subepithelial tumors. J Clin Gastroenterol 2011;45:330-336.

10. Kang JH, Lim JS, Kim JH, et al. Role of EUS and MDCT in the diagnosis of gastric submucosal tumors according to the revised pathologic concept of gastrointestinal stromal tumors. Eur Radiol 2009;19:924-934. 